
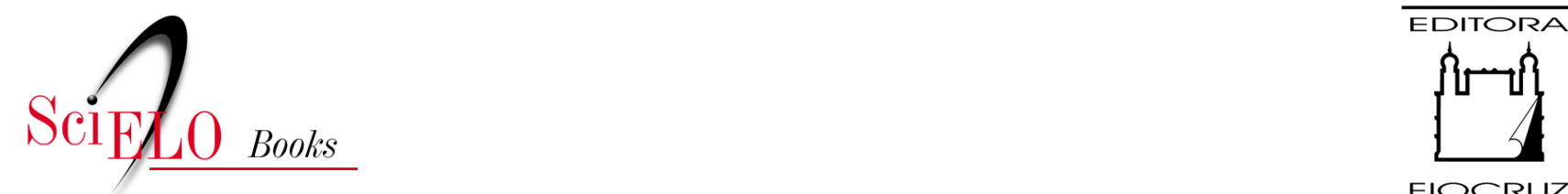

FIOCRUZ

\title{
III Anexos \\ Anexo C - Diretriz clínica para prevenção de Acidente Vascular Cerebral perioperatório em cirurgias que não sejam cardíacas, carotídeas ou neurológicas
}

\author{
Isabela Ribeiro Simões de Castro \\ Ronaldo Franklin de Miranda
}

\section{SciELO Books / SciELO Livros / SciELO Libros}

CASTRO, I. R. S., and MIRANDA, R. F. Diretriz clínica para prevenção de Acidente Vascular Cerebral perioperatório em cirurgias que não sejam cardíacas, carotídeas ou neurológicas. In: SOUSA, P., and MENDES, W., comps. Segurança do paciente: conhecendo os riscos nas organizações de saúde [online]. 2nd ed. rev. updt. Rio de Janeiro, RJ : CDEAD, ENSP, Editora FIOCRUZ, 2019, pp. 475-481. ISBN 978-85-7541-641-9. https://doi.org/10.7476/9788575416419.0024. \section{International license.}

All the contents of this work, except where otherwise noted, is licensed under a Creative Commons Attribution 4.0

Todo o conteúdo deste trabalho, exceto quando houver ressalva, é publicado sob a licença Creative Commons Atribição 4.0. 


\section{Anexo C - Diretriz clínica para prevenção de Acidente Vascular Cerebral perioperatório em cirurgias que não sejam cardíacas, carotídeas ou neurológicas}

\section{Isabela Ribeiro Simões de Castro e Ronaldo Franklin de Miranda}

Acidente Vascular Encefálico ou Cerebral (AVE ou AVC) é definido como déficit neurológico focal ou global de causa vascular cerebral, que persiste por mais de 24 horas ou seguido de morte dentro de 24 horas. Trata-se de uma importante causa de mortalidade, principalmente nos idosos.

O AVC perioperatório (durante ou até 30 dias de cirurgia não cardíaca) tem incidência que varia de 0,05 a 8\%; após cirurgias cardíacas, carotídeas ou neurológicas, essa incidência pode ser ainda mais elevada, variando de 2,2 a 5,2\%. A etiologia mais frequente é de origem tromboembólica.

A mortalidade intra-hospitalar no período perioperatório pode ser 2 a 8 vezes maior que a dos AVCs que não ocorrem no período perioperatório, provavelmente pelo atraso no diagnóstico. Vale ressaltar que as alterações inflamatórias desencadeadas nesse período ocasionam agravamento da lesão isquêmica cerebral por causa de alteração na função endotelial, induzindo à hipercoagulabilidade nesse período (Kam, Calcroft 1997).

A hipotensão arterial deve ser sempre tratada, pois é uma causa direta de AVC, provocando também aumento na lesão já existente. O estudo Poise (Devereaux 2008), ao utilizar uma dose elevada de betabloqueador no pré-operatório, ocasionou prevalência maior de hipotensão arterial e aumento na incidência de AVC no grupo que utilizou metropolol. Desse modo, devemos ser cautelosos com o uso de betabloqueador pré-operatório. A diretriz brasileira de avaliação pré-operatória (Gualandro 2011) enfatiza a importância do betabloqueador como cardioprotetor, bem como outras medidas em pacientes com isquemia miocárdica sintomática ou evidenciada por prova funcional em pacientes que já recebem betabloqueadores e, com menor evidência, os com risco cardíaco intermediário. O uso do betabloqueador não deve ser descartado pelo receio de o paciente ter um AVC. O uso do betabloqueador deve ser titulado no pré-operatório, devendo-se evitar o envio imediato para o centro cirúrgico após sua prescrição. 
A mortalidade precoce no AVC ocorre por edema cerebral e hipertensão intracraniana, e a mortalidade tardia por broncoaspiração, pneumonia, distúrbio metabólico, sepse, ou infarto/enfarte agudo do miocárdio. A maioria dos AVCs perioperatórios ocorre entre o segundo e sétimo dia de pós-operatório. Os fatores predisponentes mais consistentemente ligados ao AVC perioperatório estão listados no Quadro 1.

Quadro 1 - Fatores de risco para AVC no perioperatório

\begin{tabular}{|l}
\hline Fatores pré-operatórios (relacionados ao paciente) \\
\hline $\begin{array}{l}\text { Idade }>70 \text { anos (marcador de diminuição de reserva cerebrovascular e de coexistência de } \\
\text { patologias) }\end{array}$ \\
\hline Sexo feminino \\
\hline $\begin{array}{l}\text { História de hipertensão arterial sistêmica, diabetes mellitus, insuficiência renal (creatinina > } \\
2 \text { mg/dl), tabagismo, doença pulmonar obstrutiva crônica, doença arterial periférica, doença } \\
\text { cardíaca/doença de foro cardíaco - coronariopatia, arritmia, insuficiência cardíaca, disfunção } \\
\text { sistólica (fração de ejeção < } 40 \%, \text { principalmente nos submetidos à cirurgia de revascularização } \\
\text { miocárdica) }\end{array}$
\end{tabular}

História de AVC ou de acidente isquêmico transitório (AIT)

Estenose carotídea (em especial se sintomática)

Aterosclerose de aorta ascendente (em pacientes em pré-operatório de cirurgia cardíaca)

Parada/paragem abrupta de terapia antitrombótica prévia à cirurgia

Fatores intraoperatórios (relacionados ao procedimento)

Tipo e natureza do procedimento cirúrgico

Tipo de anestesia

Duração da cirurgia. Em procedimentos cardíacos, duração do bypass cardiopulmonar e tempo de clipagem aórtica.

Manipulação de lesões arterioscleróticas de aorta proximal

Arritmias, hiperglicemia, hipo ou hipertensão

Fatores de risco pós-operatórios

Insuficiência cardíaca, fração de ejeção baixa, infarto/enfarte agudo do miocárdio ou arritmias (fibrilação/fibrilhação atrial)

Desidratação e perda sanguínea

Hiperglicemia

Fonte: Selim (2007) 
Com relação ao tipo de cirurgia e incidência de AVC, a literatura é controversa. A presença de uma lesão carotídea assintomática pode se instabilizar no perioperatório e ocasionar um AVC; entretanto, o manuseio de uma lesão carotídea pré-operatória deve ser semelhante ao recomendado se o indivíduo não for realizar um procedimento cirúrgico (Augoustides 2012).

Há relato de menor incidência e mortalidade em pacientes submetidos à artroplastia de quadril (Mashour 2014), podendo refletir a anticoagulação precoce que geralmente ocorre nesses pacientes. Outros fatores, como cirurgia em posição sentada, administração prévia de radioterapia, rotação do pescoço, ainda não tiveram suas contribuições ao risco claramente definida, mas os cuidados nessas situações, com alterações bruscas de pressão arterial, devem ser redobrados considerando o percentual de queda em relação ao valor basal da pressão arterial mais que os números absolutos de pressão arterial a serem atingidos.

As medidas de prevenção para minimizar o risco de AVC são: cessação do tabagismo; controle da pressão arterial e manutenção da terapêutica em uso (mesmo betabloqueadores, se já vinham sendo utilizados, mas evitar começá-los no pré-operatório por período inferior a 7 dias (Foex Sear, 2014); a anticoagulação para fibrilação/fibrilhação atrial e manutenção dos antiarrítmicos em uso; administração de hipolipemiantes e terapia antiplaquetária; manejo da anticoagulação oral que vinha sendo utilizada por qualquer que seja a causa (retirar, usar anticoagulante que permita manejo com períodos mais curtos etc.); evitar alterações bruscas de glicemia. A técnica anestésica geral ou regional não é um fator de risco a ser considerado, não havendo diferença significativa que beneficie uma ou outra, nem técnicas ventilatórias específicas (Gualandro 2011 ).

\section{Medidas de prevenção em pacientes com maior risco de acidente vascular encefálico}

Nesses casos, as medidas são:

瞵 Em pacientes com história de AVC prévio: adiar o procedimento preferencialmente por três meses, otimizar a perfusão cerebral, realização de doppler transcraniano, se necessário, em razão de cirurgia em período anterior. 
锩 Em pacientes com sopro carotídeo: endarterectomia de carótidas, em paciente sintomático e assintomático, se obstrução for maior que $50 \%$ de acordo com os critérios do Nascet (Augoustides 2012).

镂 Em pacientes com fibrilação/fibrilhação atrial recente: controlar a frequência cardíaca e anticoagulação.

\section{Resumo das medidas de prevenção de acordo com o período perioperatório}

As medidas de prevenção para minimizar o risco de AVC devem variar de acordo com o período perioperatório da seguinte forma:

* No período pré-operatório: normalização da hipertensão arterial sistêmica, manutenção da glicemia < 180 e uso de estatinas em caso de hipercolesterolemia, associado com dieta (evidência 1A), interrupção de alcoolismo e tabagismo (evidência 1A). Evitar início de diurético nesse período. O uso de diurético deve ser reservado para os portadores de congestão venosa sistêmica.

踪 No período intraoperatório: controle hemodinâmico, visando especial atenção ao surgimento de hipotensão arterial que deve ser evitada. O controle glicêmico deve ser realizado tentando evitar hiper ou hipoglicemia, manutenção de normocarbia.

镂 No período pós-operatório: equilíbrio eletrolítico é de fundamental importância para se evitar o surgimento de arritmias, potencialmente emboligencias, hidratação, controle glicêmico, anticoagulação.

\section{Manuseio da anticoagulação}

O uso de anticoagulantes tem sido cada vez mais frequente, tendo em vista a profilaxia de eventos tromboembólicos. Ao avaliarmos um paciente nesse contexto, precisamos saber o risco das complicações hemorrágicas no período per e pós-operatório. O Quadro 2 descreve os riscos no manuseio do indivíduo em uso de terapia anticoagulante referente ao procedimento cirúrgico. 
Quadro 2 - A anticoagulação em função do risco de sangramento

\begin{tabular}{|c|c|c|}
\hline $\begin{array}{l}\text { Risco de } \\
\text { sangramento }\end{array}$ & Pré-operatório & Pós-operatório \\
\hline Baixo ou moderado & $\begin{array}{l}\text { - Parar HBPM que vinha sendo } \\
\text { utilizada como ponte de } \\
\text { anticoagulação em dose } \\
\text { terapêutica de manhã, 20-24 } \\
\text { horas antes da cirurgia. } \\
\text { - Omitir dose da noite se regime } \\
\text { de duas vezes ao dia. } \\
\text { - Dar } 50 \% \text { da dose total se } \\
\text { regime de uma vez ao dia. }\end{array}$ & $\begin{array}{l}\text { - Dar dose terapêutica de HBPM } 24 \\
\text { horas após a cirurgia e se houver } \\
\text { hemostasia adequada. }\end{array}$ \\
\hline Alto risco & $\begin{array}{l}\text { - Parar HBPM que vinha sendo } \\
\text { utilizada como ponte de } \\
\text { anticoagulação em dose } \\
\text { terapêutica de manhã, 20-24 } \\
\text { horas antes da cirurgia. } \\
\text { - Omitir dose da noite se regime } \\
\text { de duas vezes ao dia. } \\
\text { - Dar } 50 \% \text { da dose total se } \\
\text { regime de uma vez ao dia. }\end{array}$ & $\begin{array}{l}\text { - Dar dose terapêutica de HBPM } \\
48 \text { a } 72 \text { horas após a cirurgia e se } \\
\text { houver hemostasia adequada. } \\
\text { - Administrar dose baixa de HBPM } \\
\text { ou não fazer a medicação. }\end{array}$ \\
\hline
\end{tabular}

Fonte: Traduzido de Darvish-Kazem e Douketis (2012).

O uso de trombolítico para tratamento do acidente vascular embólico no período pré-operatório segue os mesmos critérios de inclusão e exclusão para pacientes com AVC isquêmico agudo, de três horas de início dos sintomas, conforme critérios que vêm sendo propostos pela Society for Neuroscience in Anesthesiology and Critical Care, chamado SNACC Task:

* Critérios de inclusão: diagnóstico de acidente vascular cerebral isquêmico causando déficit neurológico mensurável, início dos sintomas $<3$ horas antes do início do tratamento, $\geq 18$ anos.

* Critérios de exclusão: traumatismo craniano significativo ou AVC prévio em três meses anteriores; sintomas que sugerem hemorragia subaracnoide; punção arterial em local não compressível nos sete dias anteriores; hemorragia intracraniana anterior; neoplasia; malformação arteriovenosa ou aneurisma intracraniano; cirurgia intracraniana recente; pressão arterial elevada (sistólica > 185 $\mathrm{mm} \mathrm{Hg}$ ou diastólica > $110 \mathrm{mmHg}$ ); sangramento interno do Active; diátese hemorrágica aguda, incluindo, mas não limitado a plaquetas $<100$ 000/mm3; TTPA maior que o limite superior do normal, tendo recebido heparina nas últimas 48 horas, uso atual do anticoagulante com INR > 1,7 ou PT > 15 segundos; uso de 
inibidores diretos da trombina ou inibidores do fator Xa direto, com testes laboratoriais alterados (tais como TTPA, INR, contagem de plaquetas etc.); glicemia < $50 \mathrm{mg} / \mathrm{dL}$; CT demonstra infarto/enfarte multilobar (hipodensidade $>1 / 3$ hemisfério cerebral).

* Critérios de exclusão relativa: com cuidadosa consideração e ponderação de risco, alguns pacientes podem receber tratamento fibrinolítico, apesar de uma ou mais contraindicações relativas: sintomas pequenos ou melhorando rapidamente, gravidez, início com convulsão, cirurgia de grande porte ou trauma grave nos últimos 14 dias, hemorragia gastrointestinal ou urinária recente (nos últimos 21 dias), infarto/enfarte agudo do miocárdio recente (nos últimos três meses).

É importante que as organizações de saúde padronizem um protocolo validado para lidar com esse evento, definindo: a equipe que deverá ser acionada em caso de sinais e sintomas neurológicos, o treinamento do profissional que trabalha na instituição para reconhecer a escala de magnitude de déficit neurológico provocado pelo AVC, que será utilizada para realização do diagnóstico precoce, e a prioridade em setor de imagem para realizar uma tomografia computadorizada de crânio o mais precocemente possível. A equipe de saúde deve estar treinada para as seguintes condutas de cuidados básicos:

橉 administração do trombolítico após discussão interdisciplinar, pesando risco de sangramento;

滕 administração de aspirina, se possível (exceto nas primeiras 24 horas se for realizada trombólise);

monitorização cardíaca por pelo menos 24 horas;

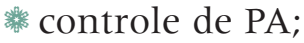

噒 uso de oxigênio para manter saturação maior que 94\%;

uso de ventilação mecânica se houver grande comprometimento ventilatório;

prevenção de complicações clínicas associadas, incluindo a solicitação de eletrocardiograma e enzimas cardíacas.

A existência de indicadores da evolução de portadores de acidente vascular encefálico pode ser utilizada para se aferir a qualidade dos serviços prestados em diferentes unidades de saúde. A seguir, são apresentados três exemplos de indicadores que podem ser utilizados no acompanhamento do atendimento prestado ao paciente: 
镂 Número de pacientes com resultados diagnósticos disponíveis para os anestesistas.

絜 Número de pacientes com sintomas instáveis tratados antes da cirurgia.

滕 Número de pacientes com complicações cardiológicas no pósoperatório (insuficiência cardíaca, AVE, IAM, arritmias).

Esses indicadores têm baixo custo de implantação e podem ser realizados em períodos menores de 12 meses. Entre as dificuldades em sua implantação estão: a coleta/recolha em múltiplas unidades, a mudança de cultura institucional e a evidência, que é pequena (nível III), disponível apenas em estudos publicados, alguns com grupo controle.

\section{Referências}

Augoustides JG. Advances in the management of carotid artery disease: focus on recent evidence. J Cardiothorac Vasc Anesth. 2012 Feb;26(1):166-71.

Darvish-Kazem S, Douketis JD. Perioperative management of patients having noncardiac surgery who are receiving anticoagulant or antiplatelet therapy: an evidence-based but practical approach. Semin Thromb Hemost 2012; 38:652-660. doi: 10.1055/s-0032-1326781

Devereaux PJ, Yang H, Yusuf S, et al. Effects of extended-release metoprolol succinate in patients undergoing non-cardiac surgery (POISE trial): a randomized controlled trial: POISE Study Group. Lancet. 2008 May 31;371(9627):1839-47. DOI: 10.1016/S0140-6736(08)60601-7.

Foex P, Sear JW. 囚-Blockers and cardiac protection: 5 yr on from POISE Br. J. Anaesth. 2014;112 (2):206-10.

Gualandro DM, Yu PC, Calderaro D, Marques AC, Pinho C, Caramelli B, et al. $2^{\text {a }}$. diretriz de avaliação perioperatória da Sociedade Brasileira de Cardiologia. Arq Bras Cardiol. 2011; 96(3 supl.1):1-68.

Kam PCA, Calcroft RM. Peri-operative stroke in general surgical patients. Anaesthesia. 1997, $52: 879-83$

Kikura M, Bateman BT, Tanaka KA. Perioperative ischemic stroke in non-cardiovascular surgery patients. J Anesth. 2010 Oct;24(5):733-8.

Mashour G. Perioperative care of patients at high risk for stroke after non-cardiac, non-neurologic surgery: guidelines from the Society for Neuroscience in Anesthesiology and Critical Care. SNACC Task. 2014 Jan 20. In comments. Draft version.

$\mathrm{Ng} J$, Chan MT, Gelb AW. Perioperative stroke in noncardiac, nonneurosurgical surgery. Anesthesiology. 2011 Oct;115(4):879-90

Selim M. Perioperative stroke. N Engl J Med. 2007 Feb 15;356 (7):706-13. 